\title{
THE SWEDISH SYSTEMS OF BONUS
}

\section{CARl Philipson,}

Sweden

1. According to the provisions of the Swedish tables of rates the premium payable on a certain policy both for Third Party Liability and for Car Damage Insurance (without clause for particular self retention) is equal to the initial premium reduced with regard to points earned as related to years with no indemnities. One such point is earned for each year during which no indemnity has been paid on the policy; upon an indemnity one or two points are lost. The detailed rules for the reduction have been altered from time to time, the existing rules have been valid from the year I952, these rules will here be called system II, while system I denotes the rules valid during the years immediately previous to 1952 . The systems are described in the following table.

\begin{tabular}{c|c|c|c|c}
\hline \multicolumn{2}{c|}{ Points lost on an indemnity } & \multicolumn{3}{|c}{$\begin{array}{c}\text { Ratio of premiums payable to } \\
\text { initial premium }\end{array}$} \\
\hline System I & System II & Points & System I & System II * \\
\hline \multirow{3}{*}{ I } & & 0 & I.00 & 1.00 \\
& & I & 0.90 & 1.00 \\
& & 2 & 0.80 & 0.70 \\
& \multirow{3}{*}{2} & 3 & 0.60 & 0.70 \\
& & 4 & 0.40 & 0.50 \\
& & 5 & 0.40 & 0.50 \\
& & 6 & 0.40 & 0.30 \\
& & 7 & 0.40 & 0.30 \\
\hline
\end{tabular}

* For Car Damage Insurance the benefits on policies with o points are reduced by $200 \mathrm{Sw}$. Cr.

2. The probabilities for the occurrence of a claim and for the occurrence of a lapse for a policy can be considered functions of two variables, the points obtained in the bonus scale, $b$, and the "duration" i.e. the time elapsed since the policyholder signed his 
first policy, $\tau$. For simplicity two kinds of models have been constructed, one assuming the probabilities to depend only on $\tau$, the other assuming the probabilities to depend only on $b$. On specifying such functions and on the assumption that the number of claims incurred are distributed in a Poisson probability distribution the distribution of a group of policies over $\tau$ and $b$ can be computed for equilibrium conditions i.e. a state for which the total number of entrants into and of exits from a bonus class-defined by the number of points-balance during a calendar year. From such distributions one may calculate the bonus factor for a given bonus system and for given values of average claim frequencies and lapse frequencies, where the bonus factor is taken to mean the quotient between the initial premium and the average premium payable of the group. It has been found that the bonus factor based on the assumption that the frequencies of claims and lapses depend only on $\tau$ very nearly coincided with the factor derived on the assumption that the frequencies depend only on $b$. It has, further, been found an approximate agreement between the factors derived on three different assumptions with respect to the variation of the claim frequency with $\tau$. The convergency of the bonus factor upon a change in the average claim frequency towards equilibrium has also been studied.

3. In several investigations such models have been compared with statistical data. As the first example a report shall be given here of an investigation for Third Party Liability and for the calendar year 1950. A second example is referred to in section 4 .

The material of $195^{\circ}$ consisted of four main groups i.e. two different areas for each cf Private Cars and Trucks of a specified category (heavy trucks, not for hire). Of the areas one referred to the two greatest cities of the country and the other to a region of the far countryside. As the registration did not with certainty state the date for underwriting the first policy of each policyholder - the date registered referred, in some cases, to the last alteration of the policy - the policies within each main group were grouped with regard to presumed duration, $\tau^{\prime}$, in five subgroups $\left(\tau^{\prime}=1,2\right.$, $3,4, \geqslant 5)$. Within each subgroup finally the risk measures were separately estimated for each of three claim classes with regard to magnitude of the amount indemnified $(<$ I.000 Sw. Cr., from 
I.000, inclusive, up to $20.000 \mathrm{Sw}$. Cr., and from $20.000 \mathrm{Sw}$. Cr., inclusive). For the last class the claim frequencies were equalized so that the frequency of the subgroups was obtained through distribution of the average for the main group in proportion to the frequencies of the middle class. This method is based on Excess Claims Analysis as described by Almer. (Trans. XVth Int. Congr. Act., New York I957, II, pp. 342-343). The total claim frequency and the risk premium of each subgroup were evaluated. The ratio between the total risk premium for $\tau^{\prime}=I$ and $\tau^{\prime} \geqslant 5$ were found to be $2.3 \mathrm{I}, 2.2 \mathrm{I}, 2 . \mathrm{I} 9,2.0 \mathrm{I}$, respectively, for the four main groups. For the purpose of comparison with a mathematical model the mean values of $\tau^{\prime}$ in the subgroups $\tau^{\prime} \geqslant 5$ were roughly estimated from the registration.

In order to compute a standard of comparison it was assumed that for $\tau=\mathrm{I}, 2, \geqslant 3$ the claim frequencies were equal to $2.5 k$, I. $k$, I.o $k$ respectively and the lapse frequencies equal to $2.0 l$, I. $l$, I.o $l$ respectively, where $k, l$ are independent of $\tau$ but assuming different values for each main group. On these assumptions. and on the assumption that the number of claims within each subgroup was distributed with a Poisson probability distribution, the distribution of the number of policies in each main group over $b$ and $\tau$ was ascertained. On the assumption that $q \%$ of the policies in each subgroup were erroneously grouped with regard to "duration" i.e. $\tau^{\prime}<\tau$ and choosing for $q$ the values 20,30 and $40 \%$ respectively three different modified distributions over $\tau^{\prime}$ and $b$ for each main group were calculated. These models were then applied to system I as defined in section I for the determination of the ratio between the premium payable for each subgroup and the average premium payable for the main group, it was found that the different sequences of these ratios obtained by the three assumptions for $q$ did not materially differ. Therefore, the sequence for $q=30 \%$ was chosen. This sequence refers to premiums loaded for administration costs, a part of these costs, in fact, not being proportionate to the risk premiums. After having made reasonable assumptions in respect of the magnitude of this non-proportionate part of the costs for administration, it was found that no appreciable error was introduced by accepting the sequence of ratios obtained as a standard of comparison for the ratio between the total risk premium 
of each subgroup and the average total risk premium of the main group.

The quotient obtained by division of the last-mentioned ratio with the corresponding ratio of the model should, then, on the hypothesis that the empirical results may be satisfactorily explained by the model, be of mean unity. The standardized deviation from this mean derived by variances of the risk premiums of the claim classes (cf. Almer, 1.c. p. 344) is on the same hypothesis approximately distributed in Student's distribution of $t$. For each of 18 subgroups of the 20 subgroups investigated the $t$-test lead to the acceptance of the hypothesis, for the remaining two groups the $t$-values were not greater than would be expected in a sequence of 20 values with a probability of $10-20 \%$. An indication for a deviation for $\tau^{\prime}=I$ is found if the quotients for each value of $\tau^{\prime}$ are averaged over the main groups using the reciprocals of the variances as weights. In fact, the possibility of a higher risk than would be expected from the model is, thus, not excluded, this deviation being particularly marked for the city area.

4. The second example is an investigation for the calendar year 1955 for Third Party Liability and for Car Damage Insurance. The data referred to Private Cars and were for each insurance form divided into three areas, one referred to the three greatest cities of the country and the other two different regions of the countryside. The statistical risk premiums were calculated with the method described in 4 , for Car Damage Insurance the claim classes were two and the calculation, therefore, accomplished without equalization. The ratio between the total risk premiums for $\tau^{\prime}=I$ and $\tau^{\prime} \geqslant 7$ were for Third Party Liability 3.02, 2.I6 and 3.62 for the three areas investigated and for Car Damage Insurance 3.92, 4.10 and 3.88 respectively. For the two comparable areas with those investigated for the year I950 the ratio, for Third Party Liability, between the total risk premiums for $\tau^{\prime}=\mathrm{I}$ and $\tau^{\prime} \geqslant 5$ were 2.6I, 3.48 against 2.3I, 2.2I, respectively, for the year I950. The slope of the curve representing the relation between the risk premium and $\tau^{\prime}$ is, thus, steeper for 1955 . This seems to be connected with a phenomenon to be discussed in section 5 .

For obtaining a standard of comparison the same methods as those described in the previous section were applied to system II, 
with respect to Car Damage Insurance regard was paid to the reduced benefits for policies with o points in the computation of the standard. For Third Party Liability the $t$-test lead to the acceptance of the hypothesis that the model gives an adequate description of the data, though also here an indication of a higher risk for $\tau^{\prime}=\mathrm{I}$ could be found, in this case more marked for one of the country regions. The agreement between the data and the standard could be improved by the substitution of a smooth curve for the broken lines in the assumed relation between claim frequency and $\tau^{\prime}$. A certain irregularity of the quotients for $\tau^{\prime}=5$ in the cities was found to be connected with a higher mean value of a claim in this group, which may be due to particular reasons. For Car Damage Insurance the $t$-test applied to subgroups with $\tau^{\prime}>$ I gave reason for accepting the model as an adequate description of the data. The risk premiums for $\tau^{\prime}=\mathrm{I}$ of Car Damage Insurance were found to be very high. There is reason to suspect that the material of this group is the result of a selection which was due to the intensification of the canvassing of the insurance without right to bonus from the year 1955, inclusive.

5. In any bonus system a policyholder may be tempted to relinquish his benefit for an accident for which the amount indemnified should be deemed to be less than his expectations of the future gains by keeping his position on the bonus scale. This involves that a minimum loss clause with a minimum amount to be arbitrarily varied by the policyholder is implied in every bonus system. This phenomenon might be called hunger for bonus. During the years before 1952 the hunger for bonus was-probably-not very marked. From the year 1952, inclusive-when the rates were materially raised and the system II was substituted for system I as defined in section I--the hunger for bonus increased materially. In fact, the claim frequency of the lowest claim class $<x$ r.000 Sw. Cr.) was on an average reduced by about $50 \%$, the form of the claim distribution function (conditioned by the occurrence of one claim) was found to change materially for values below this limit. The hunger for bonus increased also during the years after I952. The total costs for the claims will only slightly be affected by the reduction of the claim frequency as the mean amount of a claim materially increases, for $195^{2}$ the decrease of the total 
amount of the claims has been estimated to be about $5 \%$. Presumably the hunger for bonus is much more marked in respect of policyholders who already have attained several points of a given bonus scale. Thus, the steep slope in the curve representing the risk premiums in relation to $\tau^{\prime}$ for 1955 may, to a great part, be explained by the increase in bonus hunger. For a bonus system of which the curve representing the relation between the premium payable and $b$ is less steep than the corresponding curve of system II, the hunger for bonus will presumably be less marked.

6. If the bonus factor for equilibrium conditions for an average claim frequency of $p$ is used for calculating the initial premiums in a table of rates, a decrease of the claim frequency will lead to a decrease in the premium income. The increased bonus hunger has, thus, an after-effect which, in the long run, will counteract the decrease in the costs for claims caused by the increased bonus hunger. Also, for systems for which the reduction of the initial premium is restricted in comparison with the rules of system II, the bonus factor will be lower.

In older Swedish tables of rates the scale for reduction of the premium payable with regard to years with no indemnities was restricted to 30 or $40 \%$ of the initial premium. These scales were, in fact, not in conformity with the statistical relation between the risk premiums and $\tau^{\prime}$. Consequently, the premiums payable for low values of $\tau^{\prime}$ were insufficient. Considering a stock of policies underwritten in a certain calendar year the company then had to reckon with a substantial loss during this year and some years thereafter. This loss was calculated to be equalized by the profits of later years. In the actuarial technique these facts implied the difficulties of negative reserves during the first years of the insurance. The implications of negative reserves are known from the gross reserve technique of life insurance, though the negative reserves are in this technique mostly due to the distribution of administration costs rather than to the risk itself. Furthermore, it was found that the older bonus systems lead to irrational transfer of older policies from company to company by competition. The economic results of the whole business depended under these older bonus systems, to a great extent, on the frequency of underwriting and lapses during the year of account. Especially during the 
second world war and the years after the peace treaty, these frequencies showed material variation due to rationing of gasoline and ceasing of the rationing. The effects on the economic results were very confusing. During the war the funds of Third Party Liability increased in proportion to the stock insured so that it was found appropriate to refund a part of the funds to the policyholders. In the years after the war the increased underwriting lead to material losses. which forced the companies to a material increase in the rates on several occasions. An analysis has shown that a great part of these variations could be traced back to the properties of the bonus systems then in existence.

7. In recent years some students have criticized the system II by several arguments. Firstly the existence of a particular group of policyholders with aggravated risk should disturb the relations so as to render a system based on the statistics irrational. Furthermore, the high initial premiums prevent the companies from extending the voluntary Car Damage Insurance with no particular self retention clause to a greater group of car owners. Statistical investigations of recent years seem to indicate that the dependence on "duration" is much more complicated than could be inferred from the investigations described above. So far such investigations carried out by different companies seem-to a certain extentto be contradictory. According to the author's opinion one prominent cause of this complexity may be found in the selection achieved by the competition between the insurance with right to bonus, without particular self retention and that without right to bonus, with particular self retention. An argument often appearing in the discussions is that the principle of equity demands that old sinners shall get a just punishment, harder than that imposed on new policyholders with unknown risk. To the author's opinion it is difficult to argue for just treatment as this has to be based on the occurrence of claims which-also for good risks-is subject to heavy chance fluctuations.

8. It should here be mentioned that the author has in an internal paper described various modifications of the present system. It has been found that a simplified scale for the reduction of the premiums payable combined with a scale of increasing self retention on the claims, after the first claim in the group where the policies 
have scored no points $b$, the reduction of the initial premium will as a rule be only a few per cent, in one modification 7 per cent. A change of the premium level for the better risks seemed not to be appropriate.

The modifications involve two advantages, one is a better equalization over time, the other the psychological effect of the punishments for repeated claims. The suggested modifications with an increasing self retention for several claims leads to easier administration than the Norwegian system which is based on additional premiums for several claims. In the latter case a control register must be consulted for every transfer of the policy from a company to another, in the suggested form such a reference has only to be made if a claim occurs in the year of transfer.

If the modified system gives approximately the same premium levels for all durations as system II i.e. in conformity with the variation of the risk the disadvantages of the older systems with restricted reduction of premiums can be avoided. The tables for Car Damage Insurance ought to be rationalized by a restriction of the choice of the particular self retention to one standard amount of every risk group. The adaptation to the risk is improved by the scale of increasing amount of a compulsory self retention on several claims. It is a question of judgement whether the necessary implications in the routine for settlement of claims shall be taken in order to achieve such an improvement of adaptation. The problem seems to be on the border between the principle of equity and that of solidarity. 